\title{
De la nature réflexive de la sociologie et de la disparition de son objet
}

Jacques Hamel

\section{OpenEdition}

\section{Journals}

Édition électronique

URL : http://journals.openedition.org/ress/185

DOI : $10.4000 /$ ress. 185

ISSN : 1663-4446

Éditeur

Librairie Droz

Édition imprimée

Date de publication : 1 novembre 2007

Pagination : 45-56

ISBN : 978-2-600-01207-2

ISSN : 0048-8046

Référence électronique

Jacques Hamel, « De la nature réflexive de la sociologie et de la disparition de son objet », Revue européenne des sciences sociales [En ligne], XLV-139 | 2007, mis en ligne le 01 novembre 2010,

consulté le 01 mai 2019. URL : http://journals.openedition.org/ress/185; DOI : 10.4000/ress.185 


\title{
Jacques HAMEL
}

\section{DE LA NATURE RÉFLEXIVE DE LA SOCIOLOGIE ET DE LA DISPARITION DE SON OBJET}

\begin{abstract}
RÉSUMÉ
Cet article aborde la question de la réflexivité de la sociologie. Après avoir brièvement retracé la nature réflexive de l'explication sociologique, l'auteur se penche sur les méthodes de l'intervention sociologique (A. Touraine) et de l'auto-analyse provoquée et accompagnée (P. Bourdieu) afin d'en saisir les modalités. Le statut conféré au sens commun et la conception de l'analyse sont également envisagés dans cette voie. A l'ère de la modernité, la réflexivité de la sociologie contribue à la mutation de son objet en un sujet capable de créer son identité et sa position sociale de son propre chef. Estce à dire que le glas a sonné pour la sociologie vouée à expliquer à la lumière des «déterminations sociales » puisque son objet disparaît dans ce contexte?
\end{abstract}

\section{ABSTRACT}

This article explores the question of reflexivity in sociology. After briefly tracing the reflexive nature of sociological explication, the author looks at the methods of sociological intervention (A. Touraine) and provoked and accompanied self-analysis (P. Bourdieu) in an attempt to understand how they work. The status of common sense and the design of analysis are also considered from this perspective. In the modern era, the reflexivity of sociology plays a role in transforming its object into a subject that is capable of creating its own identity and social position. Does this spell the end for a sociology dedicated to providing explanations in the light of «social determinations » as its object disappears in this context?

La nature réflexive de la sociologie fait actuellement l'objet de vifs débats. La réflexivité semble être à bien des égards la notion à l'ordre du jour de la discipline. Les considérations de toutes sortes abondent sur le sujet. En effet, de nos jours les sociologues cherchent plus que jamais à concevoir la théorie sous la forme de l'explication propre à éclairer directement la société et, au-delà, la vie des individus qui gravitent en son sein.

La question des retombées pratiques de la théorie sociologique n'a certes rien de neuf. L'ingénierie sociale en vigueur dans la sociologie américaine veut, depuis les débuts de l'Ecole de Chicago, donner à la sociologie «les mêmes niveaux de fiabilité dans l'action que celles des sciences de l'ingénieur ${ }^{1}$ dont l'industrie offre le «modèle de rationalité». Sous d'autres obédiences, nombreux sont les sociologues à s'être ouvertement interrogés sur la capacité de leur théorie à faire office de vecteur propre à infléchir la vie des sociétés et de celle de leurs membres. A la belle époque du marxisme triomphant, la théorie « révolutionnaire», produite

1 Suzie Guth, Chicago 1920, aux origines de la sociologie qualitative, Paris, Taraèdre, 2005, p. 53. 
par les philosophes et les sociologues, devait d'emblée réunir les conditions propices au Grand soir et, par la suite, encourager les «masses » à garder le cap.

Sans vouloir remonter aux premières manifestations de cette tendance qui, aujourd'hui, s'affirme comme volonté ferme, Durkheim, par exemple, ne cherchait pas à son époque d'autre visée pour la sociologie quand, avec nuances, il concevait ses artisans comme des «conseilleurs » voués à créer les solidarités collectives susceptibles de donner sa bonne santé à la société. En effet, il ne faisait pas mystère qu'à ses yeux les sociologues «sont faits pour aider leurs contemporains à se reconnaître dans leurs idées et dans leurs sentiments beaucoup plus que les gouverner $»^{2}$. Sur cette base, il assignait à la sociologie une vocation éducative, celle d'éclairer à la lumière de la théorie qui, en essaimant, allait prendre tout son éclat et d'orienter la société dans la bonne voie après avoir instruit l'une et l'autre des parties sociales en présence.

\section{LA SOCIOLOGIE \\ A L'HEURE DE LA SOCIÉTÉ POSTMODERNE}

A l'ère des sociétés modernes, voire postmodernes, il ne fait plus de doute que la théorie sociologique a valeur réflexive. Force est, en effet, de constater que les explications avancées par les sociologues, de nos jours, répercutées par les médias et la culture de masse, s'inscrivent dans la réflexion que les individus formulent à propos de ce qu'ils sont et de ce qu'ils font à leur échelle. En d'autres termes, les connaissances élaborées en sociologie s'introduisent dans ce que celle-ci prend pour objet. Anthony Giddens note pertinemment à ce sujet que les théories sociologiques «vont et viennent» continuellement par rapport à leur objet: elles restructurent ce dernier, qui a de son côté appris à penser en termes de socio$\operatorname{logie} »^{3}$.

Selon lui, la modernité est de ce fait «profondément et intrinsèquement sociologique » pour la raison que, de nos jours, la théorie de cet ordre se mêle d'office à la connaissance de tout un chacun qui, par ricochet, infléchit la société dans la perspective que renferme l'explication sociologique. Les théories sociologiques ont donc une valeur réflexive plus puissante que les connaissances théoriques produites en science - comme en physique - puisque, tôt ou tard, elles deviennent le sens commun et permettent aux individus et aux groupes de «mieux se connaître». Elles contribuent dans cette voie au développement de la réflexivité conçue comme «l'examen et la révision constante des pratiques sociales, à la lumière des informations nouvelles concernant ces pratiques mêmes, ce qui altère constitutivement leur caractère $»^{4}$.

L'intimité et la sexualité se révèlent aux yeux de Giddens le terrain le plus actif de la réflexivité de la sociologie puisque l'une et l'autre ont été profondément modelées par les connaissances cumulées dans son orbite, comme du reste dans

\footnotetext{
2 Emile Durkheim, «L'élite intellectuelle et la démocratie », Revue bleue, $5^{\mathrm{e}}$ série, tome 1, 1906, p. 706.

3 Anthony Giddens, Les conséquences de la modernité, Paris, L’Harmattan, 1994, p. 49.

4 Ibid, p. 45.
} 
les autres domaines des sciences sociales. Des entreprises comme le Rapport Kinsey, par exemple, qui «à l'origine avait exactement le même but que toutes les recherches en sciences sociales: celui d'analyser ce qui se passait dans un secteur spécifique de l'activité sociale» ont trouvé un écho dans la société en étant matière à de larges débats, lesquels, en faisant boule de neige, "contribuèrent à modifier en profondeur les vues de tout un chacun sur l'activité sexuelle ainsi que sur les rapports amoureux» de sorte qu'on est fondé à penser que «l'incessant développement de ce type d'investigation sociale signale, en même temps qu'il rend possible, une réflexivité accrue au niveau des pratiques sexuelles les plus ordinaires et quotidiennes $»^{5}$.

La sociologie est donc continuellement en passe de prendre valeur réflexive puisqu'ils sont légion les individus qui rendent raison de ce qu'ils sont et de ce qu'ils font à la lumière de la théorie produite en son nom, expurgée au besoin de ses concepts et traduite en termes accessibles d'office.

Le pouvoir de la théorie sociologique en cette matière est toutefois mis en question. Les sociologues apparaissent dans ces conditions comme des experts qui font courir à leur discipline le risque qu'Ulrich Beck associe à la société du même nom: celui d'imposer des directions sociales au service des pouvoirs qui tendent de plus en plus à cacher leur vrai visage. Selon la vision de Jürgen Habermas $^{6}$, la théorie critique que représente la sociologie se mue ainsi en techniques de contrôle social funeste pour l'opinion publique ouverte au débat éclairé que le penseur allemand associe à la communication exempte de toutes contraintes ${ }^{7}$. La théorie critique, en « revendiquant un caractère scientifique » et en apparaissant sous le mode technique, échappe à «l'analyse comme à la conscience que pourrait en prendre l'opinion publique » et fait figure d'idéologie ${ }^{8}$.

Or, sans être aveugle sur la possible orientation que représente le contrôle social, souvent assimilé à la technocratie, on cherche de nos jours à concevoir positivement la réflexivité à laquelle la théorie sociologique donne corps, de manière à permettre à la sociologie de faire peau neuve. Sociologue aguerri, Alain Touraine voit poindre dans la foulée un «nouveau paradigme pour comprendre le monde d'aujourd'hui »? .

En effet, la nature réflexive de la théorie sociologique, aujourd'hui plus qu'hier exhibée par ses auteurs, se révèle propice à ce que l'acteur social se mue en un sujet conscient de «rechercher la création de soi-même au-delà de toutes les situations et fonctions ${ }^{10}$ en société, c'est-à-dire capable «d'acquérir et de manifester une conscience de soi-même autofondée ${ }^{11}$, bref de pouvoir se représenter à lui-même «sa propre capacité de création, de réflexion, et d'évaluation » ${ }^{12}$.

\footnotetext{
5 Anthony Giddens, La transformation de l' intimité, Paris, Le Rouergue / Chambon, 2004, p. 44.

6 Jürgen Habermas, La technique et la science comme «idéologie», Paris, Gallimard, 1973.

7 Jürgen Habermas, Idéalisations et communication, Paris, Fayard, 2006.

8 Jürgen Habermas, La technique et la science comme «idéologie», op. cit., p. 34.

9 Alain Touraine, Un nouveau paradigme. Pour comprendre le monde d'aujourd' hui, Paris,
} Fayard, 2005.
10 Ibid., p. 152.
${ }^{11}$ Ibid., p. 157.
${ }^{12}$ Ibid., p. 201. 
Voilà le rôle que doivent jouer les théories formulées sous l'égide de la sociologie: octroyer à l'individu la capacité de comprendre ce qu'il est et ce qu'il fait avec, à la clé, la ferme intention de devenir autonome au gré de motivations parfaitement éclairées par la théorie sociologique. Sous ce chef, l'analyse sociologique a pour but de «faire apparaître le sujet enfoui dans l'acteur social ${ }^{13}$ en le révélant sans ambages à ses propres yeux.

Avec audace, Touraine s'est employé à concevoir la méthode qui, au nom de l'intervention sociologique, donne son véritable visage à la réflexivité que soustend la théorie sociologique. Sa mise en œuvre constitue effectivement l'intervention de sociologues dans une action collective afin que celle-ci, éclairée par la théorie, puisse se muer en mouvement social. L'intervention sociologique, créée voilà trente ans, découle directement de la théorie de l'action proposée par son instigateur. En effet, Touraine conçoit en théorie la société comme l'action associable dans son esprit au pouvoir de décider de la «gestion» des ressources de toutes natures produites sous son égide - qu'elle exerce sur elle-même pour prendre corps et qui, fondamentale, devient d'emblée l'objet d'un conflit ouvert entre acteurs sociaux désireux de l'axer dans le sens de leurs orientations culturelles et éthiques établies sous la forme d'un projet politique.

Si un «acteur social» - tour à tour associé chez Touraine aux ouvriers, aux étudiants, aux féministes, aux écologistes et aujourd'hui aux gays et lesbiennes s'emparait de ce levier et l'orientait dans le sens de la vision sociale qui gouverne son action collective, celle-ci se muerait en mouvement social. Une telle mutation s'opère sous la combinaison de trois principes qui correspondent en théorie à 1) l'identité, la capacité de l'acteur collectif à se reconnaître comme acteur social apte à changer la société dans son ensemble; 2) l'opposition, la capacité d'identifier son ou ses «adversaires » qui, en affichant une autre vision sociale, vont à son encontre et 3) la totalité, la capacité de concevoir l'enjeu sous ce rapport afin de former son action en conséquence.

L'intervention sociologique, on le comprendra sans difficulté, a pour but d'alimenter l'action collective d'un acteur social afin que celle-ci se transforme en mouvement social du fait que, par ce moyen, elle donne le cap à la société dans son ensemble. Les difficultés des acteurs collectifs à s'incarner comme instigateurs de mouvements sociaux conduisent aujourd'hui Touraine à concevoir plus largement la réflexivité en attribuant à la théorie sociologique le pouvoir d'éclairer l'acteur - individu ou groupe - afin que celui-ci puisse devenir le sujet qu'il ne parvient pas à être du fait que sa capacité réflexive et créatrice devient «inconsciente». Le sujet se replie sous une forme inconsciente, sans toutefois être refoulé, au sens qu'a ce terme en psychanalyse. Il s'éclipse parce que lui font barrage «la quotidienneté, les normes de la vie publique, l'urgence des décisions pratiques, l'intensité des émotions et la recherche de l'intérêt ou de la solution à un problème difficile ${ }^{14}$. Bref, les capacités créatrices et réflexives qui donnent aux acteurs leur qualité de sujet sont recouvertes par la banalité du moi et la nature routinière de l'action à leur échelle.

\footnotetext{
${ }^{13}$ Ibid., p. 199.

${ }^{14}$ Ibid., p. 198.
} 


\section{LA MÉTHODE \\ DE L'INTERVENTION SOCIOLOGIQUE}

L'intervention sociologique s'élabore conséquemment sous la forme d'une analyse expressément destinée à faire jaillir de l'acteur les capacités créatrices et réflexives génératrices de l'autonomie propre à le constituer en sujet. Dans cette perspective, elle consiste en l'auto-analyse du conflit social de l'heure que vont entreprendre ses figures de proue en compagnie d'une équipe de sociologues et cela dans le cadre d'une série de rencontres expressément destinées à formuler sur le vif la théorie susceptible de l'expliquer et à en vérifier la justesse si l'explication sur laquelle elle débouche est avalisée par les parties en présence.

L'intervention sociologique se révèle dans cette voie «moins la mise en œuvre pratique d'une conception de la société que la conception d'un acteur dont on suppose qu'il est partiellement capable de connaissance, de réflexivité ${ }^{15}$. Elle se révèle par conséquent «une méthode qui repose sur une procédure démocratique puisqu'elle reconnaît d'emblée aux acteurs le statut de sujet ${ }^{16}$.

La méthode, on le constate, s'appuie incontestablement sur la réflexivité dont font preuve les acteurs sociaux puisque ceux-ci contribuent à l'élaboration de la théorie sous l' «intervention» des sociologues qui leur apprennent à en saisir sur le vif la valeur réflexive et, de là, à connaître la manière de la concevoir en offrant en exemple leur propre mode de réflexion. L'intervention sociologique ne manque pas d'audace à cet égard du fait qu'elle leur donne voix à ce chapitre. En effet, l'équipe des sociologues a le devoir de soumettre la théorie à l'épreuve et s'il y a conversion, adhésion des acteurs à celle-ci, l'explication qu'elle renferme se vérifie à chaud.

Les acteurs sociaux sont donc ici envisagés selon un tout nouveau statut. On leur reconnaît en effet une réflexion qui a son intérêt, voire sa valeur, pour élaborer directement la théorie sociologique. Selon François Dubet, les acteurs sociaux disposent en réalité de la «vraie connaissance de leur action ${ }^{17}$ et les sociologues doivent s'obliger à en tenir compte du fait qu'ils ont affaire en définitive à un «acteur épistémique» capable de réfléchir et de produire la connaissance de son action. Dubet renchérit en soulignant que cette perspective «invite à considérer chaque individu comme un «intellectuel», comme un acteur capable de maîtriser consciemment, dans une certaine mesure en tout cas, son rapport au monde $»^{18}$. L'intervention sociologique ne peut par conséquent être une méthode de «recueil d'opinions, c'est aussi une discussion dans laquelle le chercheur élabore ses propres thèses et, dans une certaine mesure, discute avec un «collègue ${ }^{19}$ qui prend le visage de l'acteur social.

15 François Dubet, Sociologie de l'expérience, Paris, Seuil, 1994, p. 240.

${ }^{16}$ François Dubet, «Plaidoyer pour l'intervention sociologique», dans Didier Vrancken et Olgierd Kuty (sous la dir.), La sociologie et l'intervention, Bruxelles, De Boeck, p. 108.

${ }^{17}$ François Dubet, François (1988), Acteurs et sociologues. Le cas de l' intervention sociologique, Paris, Ecole des hautes études en sciences sociales, 1988, p. 12 (miméographié).

${ }^{18}$ François Dubet, Sociologie de l'expérience, op. cit., p. 105.

${ }^{19}$ Ibid., p. 235. 
Toutefois, la réflexion des acteurs sociaux se révèle «limitée », selon Dubet, du fait que les «dimensions du système social» ou les «conditions sociales de l'action » échappent à leur conscience et deviennent par conséquent inconscientes pour la raison, semblable à celle invoquée précédemment par Touraine, que «les acteurs ont une connaissance pragmatique et causale des enchaînements de l'action, des situations, des décisions et des choix, des anticipations $»^{20}$.

La théorie sociologique a précisément pour but de les déceler et de les exhiber aux yeux des acteurs sociaux en prenant sous ces conditions valeur réflexive. Dans la perspective tourainienne, l'exercice cherche plus exactement à débusquer, dans la réflexion des acteurs sociaux, les trois principes dont la mise en œuvre, on l'a vu, permet la mutation de l'action collective en mouvement social: l'identité, l'opposition et la totalité. Si la découverte de ces trois principes apparaît à l'ordre du jour, force est de constater que, dans l'intervention sociologique, on voit difficilement comment s'opère le jeu entre la connaissance des acteurs sociaux et la théorie sociologique susceptible de donner à cette dernière sa valeur réflexive.

\section{L’AUTO-ANALYSE PROVOQUÉE ET ACCOMPAGNÉE}

La sociologie française a été témoin d'une entreprise similaire à l'intervention sociologique et susceptible, comme cette dernière, de donner à la théorie sociologique l'éclat de la réflexivité. En effet, Pierre Bourdieu s'est également employé à concevoir une méthode, l'«auto-analyse provoquée et accompagnée», capable d'étudier la Misère du monde $e^{21}$ en permettant aux individus de comprendre la position sociale qui explique l'une et l'autre figures de la misère dont ils sont victimes.

Cette méthode, à l'instar de l'intervention sociologique, cherche également à orchestrer une auto-analyse au gré d'un entretien sociologique expressément conçu pour conférer sa valeur réflexive à la théorie sociologique. La neutralisation de la violence symbolique que peut engendrer la relation enquêteur-enquêté se recommande de cette intention.

Sans qu'il n'y paraisse, le face-à-face que requiert l'entretien sociologique peut générer des distorsions dans la communication liées à l'inégalité sociale entre l'enquêteur et l'enquêté. En effet, l'échange dans ce cadre est loin de se dérouler sur un pied d'égalité puisque l'un et l'autre sont dotés de propriétés sociales différentes. En sa qualité de sociologue, l'enquêteur est par exemple universitaire, richement pourvu de titres et de ressources scolaires par contraste avec l'enquêté qui peut en être singulièrement dépourvu. Les façons d'être, de penser et d'agir de l'un et l'autre peuvent également différer à bien des égards sur cette base.

Ces différences s'expriment dans la théorie de Bourdieu en termes de capital et d' «habitus». Le capital désigne, dans cette optique, l'ensemble des ressources et des pouvoirs dont sont dotés et qu'acquièrent les individus sous différentes «espèces »: économique, culturelle, scolaire, politique, linguistique, etc. La notion d'habitus, quant à elle, correspond brièvement aux «schémas mentaux de perception, d'appréciation et d'action ${ }^{22}$ en fonction desquels les individus mobi-

\footnotetext{
${ }^{20}$ Ibid., p. 225.

${ }^{21}$ Pierre Bourdieu, (sous la dir.), La misère du monde, Paris, Seuil, 1993.

22 Pierre Bourdieu (avec la coll. de L. Wacquant), Réponses, Paris, Seuil, 1992, p. 24.
} 
lisent le capital sous leur propriété et dont le jeu détermine du coup leur position sur l'échiquier social qui se nomme champ dans le vocabulaire théorique de cet auteur.

La société se représente dans cette voie en tant que configurations de points unis par des «relations objectives» qui deviennent «justiciables d'une analysis situs» ${ }^{23}$ ou d'une géométrie sociale. Sous cette perspective, «les agents sociaux, et aussi les choses en tant qu'elles sont appropriées par eux, donc constituées comme propriétés, sont situés en un lieu de l'espace social, lieu distinct et distinctif qui peut être caractérisé par la position relative qu'il occupe par rapport à d'autres lieux (au-dessus, au-dessous, entre, etc.) et par la distance (dite parfois « respectueuse »: e longinquo reverentia) qui le sépare d'eux $»^{24}$.

La différence des positions sociales entre les interlocuteurs en présence dans l'entretien sociologique peut se traduire par l'imposition du point de vue de l'enquêteur sur celui de l'enquêté sous le mode de la violence symbolique propre à rendre cette inégalité sociale «naturelle», «évidente», bref sous une forme qui ne peut être mise en cause. L'enquêteur se prive ainsi de connaître véritablement le point de vue de son vis-à-vis et ce fait handicape sérieusement la théorie sociologique puisque celle-ci s'élabore sans tenir compte de sa réflexion. Afin d'enrayer la violence symbolique, Bourdieu joue d'astuce en proposant de mettre face à face un enquêteur et un enquêté qui correspondent à une position sociale équivalente, déterminée en théorie sous l'angle du capital et de l'habitus qui donnent corps à la géométrie sociale chère à cet auteur.

Selon lui, l'enquêteur se révèle ainsi sur un pied d'égalité avec l'enquêté en matière sociale et, de ce fait, il ne risque pas de lui imposer, inconsciemment ou non, le point de vue qui correspond à sa position dans la société. L'entrevue s'établit dans ces conditions sous le signe de l'objectivité puisque, si l'enquêteur partage avec l'enquêté «la quasi-totalité des caractéristiques capables de fonctionner comme des facteurs explicatifs majeurs de ses pratiques et de ses représentations, et auquel il est uni par une relation de profonde familiarité, ses questions trouvent leur principe dans ses dispositions objectivement accordées à celles de l'enquêté; les plus brutalement objectivantes d'entre elles n'ont aucune raison d'apparaître comme menaçantes ou agressives parce que son interlocuteur sait parfaitement qu'il partage avec lui l'essentiel de ce qu'elles amènent à livrer et, du même coup, les risques auxquels il s'expose en le livrant $»^{25}$.

L'objectivité née dans ces conditions apparaît d'autre part favorable à ce que l'enquêteur considère la réflexion de l'enquêté en reconnaissant en acte l'habitus et le capital qui leur sont communs et qu'il peut dès lors concevoir en théorie, celle de la géométrie sociale. Bourdieu note à ce sujet que «l'enquêteur ne peut oublier qu'en objectivant l'enquêté, il s'objective lui-même ${ }^{26}$ et, inversement, l'enquêté peut s'objectiver en prenant conscience de sa position sociale que lui reflète l'enquêteur. L'entretien conçu dans cette veine revêt donc une valeur réflexive puisque si l'enquêteur fait preuve d'objectivité en percevant dans l'interlocuteur

\footnotetext{
${ }^{23}$ Pierre Bourdieu, Méditations pascaliennes, coll. Liber, Paris, Seuil, 1997, p. 161.

${ }^{24}$ Ibid., p. 161.

${ }^{25}$ Pierre Bourdieu, (sous la dir.), La misère du monde, op.cit., p. 908.

${ }^{26}$ Idem.
} 
sa position sociale, par ricochet, l'enquêté peut saisir la sienne puisqu'elle se trouve réfléchie par l'enquêteur qui lui apprend à la décoder sur le vif.

Faisant état de ses entretiens sur la Misère du monde, Bourdieu souligne à cet égard que «les enquêtés, surtout parmi les plus démunis, semblent saisir cette situation [l'entretien sociologique] comme une occasion exceptionnelle qui leur est offerte de témoigner, (...) de s'expliquer, au sens le plus complet du terme, c'est-à-dire de construire leur point de vue sur eux-mêmes et sur le monde et de rendre manifeste le point, à l'intérieur de ce monde, à partir duquel ils se voient eux-mêmes et voient le monde, et deviennent compréhensibles, justifiés, et d'abord pour eux-mêmes $»^{27}$.

En d'autres termes, les enquêtés tirent bénéfice de l'exercice en prenant acte de leur position sociale et du point de vue qui lui correspond sous le mode de la géométrie sociale née de la théorie induite dans le feu de l'entretien sociologique par l'enquêteur et répercutée par sa position sociale qui fait en quelque sorte office de miroir propre à la réfléchir.

L'auto-analyse provoquée et accompagnée a connu des ratés sur ces points. En effet, Bourdieu a été forcé de constater que chercher à mettre sur un pied d'égalité l'enquêteur et l'enquêté en termes de position sociale crée une «familiarité » qui ruine à bien des égards la richesse de l'entretien. Sous ces conditions, celui-ci se déroule sans besoin que l'un et l'autre explicitent leurs propos respectifs du fait qu'ils correspondent au «point de vue» de leur commune position sociale dont ils prennent acte dans le déroulement de l'entretien sociologique.

$\mathrm{Si}$, d'autre part, l'enquêté peut prendre conscience de sa position sociale du fait qu'elle lui est réfléchie par l'enquêteur, le point de vue qu'il développe à son sujet ne correspond en rien à la théorie née de l'analyse sociologique orchestrée à tous égards par l'enquêteur acquis au métier de sociologue. L'entretien sociologique peut donc être difficilement conçu comme auto-analyse, encore moins comme analyse associée à ce que désigne ce dernier mot sur le plan épistémologique: une connaissance théorique et, par conséquent, formulée en termes abstraits. L'enquêté peut certes découvrir sa position sociale grâce à cet exercice réflexif, mais il ne peut pas l'expliquer sous la forme de la connaissance sociologique qui chez Bourdieu se formule notamment au moyen des notions d'habitus et de capital propres à produire la théorie qu'est la géométrie sociale.

Les problèmes de l'auto-analyse provoquée et accompagnée n'ont toutefois pas empêché Bourdieu de nuancer singulièrement sa conception à propos du statut conféré au point de vue des individus qui gravitent dans l'orbite sociale. Dans le Métier de sociologue, il associait cette notion au sens commun conçu péjorativement comme «représentations schématiques et sommaires qui sont formées par la pratique et pour elle, et qui tiennent leur évidence et leur autorité des fonctions sociales qu'elles remplissent $»^{28}$ et qui font que « dès qu'il réfléchit sur sa pratique, l'[individu] perd toute chance d'exprimer la vérité de sa pratique ${ }^{29}$.

\footnotetext{
${ }^{27}$ Ibid., p. 915.

${ }^{28}$ Pierre Bourdieu, Jean-Claude Chamboredon et Jean-Claude Passeron, Le métier de sociologue, Paris, Mouton, 1968, p. 28.

${ }^{29}$ Pierre Bourdieu, Le sens pratique, Paris, Minuit, 1980, p. 152.
} 
Le sens commun revêt ce statut pour la raison — pratiquement identique à celle de Touraine et de Dubet - que les individus rendent compte de leur situation en termes d'événements, d'individus, de contextes et d'autres réalités substantielles que Bourdieu associe au sens pratique formulé grâce au «langage de la familiarité» qui, répétitif ou routinier, «ne connaît que les cas particuliers et les détails de l'intérêt pratique ou de la curiosité anecdotique, parlant toujours par noms propres de personnes ou de lieux $»^{30}$. La théorie, sous l'optique de la géométrie sociale, l'explique pour sa part sous la forme de «relations objectives», «largement indépendantes des volontés et des consciences individuelles ${ }^{31}$, qui forment la position de l'individu propre à éclairer la domination sociale qui pèse sur lui en matière de ressources et de pouvoirs associables au capital.

Son enquête sur la Misère du monde, malgré ses failles, a appris à Bourdieu que les individus n'ont pas et ne peuvent avoir «la «science infuse» de ce qu'ils sont et de ce qu'ils font; plus précisément, ils n'ont pas nécessairement accès au principe de leur mécontentement ou de leur malaise ${ }^{32}$. En effet, Bourdieu n'a pas tardé à constater qu'ils cherchent à rendre raison de leur misère à la lumière des «routines de la pensée ordinaire du monde social, qui s'attache à des «réalités » substantielles, individus, groupes, etc. plus volontiers qu'à des relations objectives que l'on ne peut ni montrer ni toucher du doigt et qu'il faut conquérir, construire et valider par le travail scientifique $»^{33}$.

Les individus affichent donc une connaissance issue de leur «pratique » et qui, de nature routinière, se formule conséquemment dans ce rayon sous la forme de «réalités substantielles». Ils ont donc à l'esprit une connaissance pratique que Bourdieu qualifie joliment de «routines de la pensée ordinaire».

A ce propos, il ajoute que, dans ces conditions, la sociologie se révèle en définitive «connaissance d'une connaissance» ${ }^{34}$. Les sociologues ont donc pour métier d'élaborer des théories explicatives sur la base de la connaissance pratique des individus qui, elle, s'élabore sous la forme de routines de pensée qu'ils doivent obligatoirement déchiffrer. En d'autres termes, la géométrie sociale susceptible d'éclairer leur position puise dans la connaissance pratique des individus les «routines de pensée » requises pour établir le jeu du capital et de l'habitus propre chez Bourdieu à faire apparaître les «relations objectives » conçues comme explication sociologique.

Sous l'égide de la «connaissance de la connaissance» qu'est la sociologie, la connaissance pratique qu'affichent les individus contribue, comme on vient de le constater, à la formulation de la théorie, mais cette dernière peut inversement enrichir leur «point de vue», ainsi que Pierre Bourdieu a tenté de le faire au moyen de l'auto-analyse provoquée et accompagnée dans l'optique qu'il défendait bien avant sa mise au point, celle de donner des «armes» aux individus afin qu'ils

${ }^{30}$ Ibid., p. 153. Voir également Pierre Bourdieu, «Introduction», dans Pierre Bourdieu (sous la dir.), Un art moyen. Essai sur les usages sociaux de la photographie, Paris, Editions de Minuit, 1965, p. 17-28.

${ }^{31}$ Pierre Bourdieu (avec la coll. de L. Wacquant), Réponses, op. cit., p. 72.

32 Pierre Bourdieu, (sous la dir.), La misère du monde, op.cit., p. 918-919.

${ }^{33}$ Pierre Bourdieu, Raisons pratiques, Paris, Seuil, 1994, p. 9.

${ }^{34}$ Pierre Bourdieu (avec la coll. de L. Wacquant), Réponses, op. cit., p. 103. 
puissent renverser la domination sociale dont ils sont l'objet et bénéficier ainsi d'une «marge de liberté ${ }^{35}$.

\section{DE L'OBJET AU SUJET DE LA SOCIOLOGIE}

De par sa nature réflexive, la sociologie vient ainsi donner à la connaissance pratique des individus la touche de la théorie apte à changer opportunément ce qu'ils sont et ce qu'ils font dans le rayon de la société et sous le coup de la domination qui s'exerce à cette échelle. La modernité, on l'a vu chez Giddens, s'est à son avis largement formée au gré de l'induction des théories sociologiques dans la réflexion individuelle et collective. La sociologie, comme les autres sciences sociales fournissent ainsi aux individus les connaissances requises pour qu'ils deviennent responsables de ce qu'ils sont et de ce qu'ils font en société. Elle contribue de la sorte à l'«individualisation de la vie sociale» qu'Ulrich Beck associe étroitement à la «décomposition et à l'abandon des modes de vie de la société industrielle (classe, strate, rôle sexué, famille)» pour ceux sur la base desquels «les individus construisent, articulent et mettent en scène leur propre trajectoire individuelle ${ }^{36}$ à la lumière de leur connaissance de la vie sociale. La société devient ainsi «liquide» puisque, aux yeux de Zygmunt Bauman, «les conditions dans lesquelles ses membres agissent changent en moins de temps qu'il n'en faut aux modes d'action pour se figer en habitudes et en routines ${ }^{37}$.

En d'autres termes, les parcours biographiques des individus en société «deviennent «autoréflexifs»; ce qui était le produit de déterminations sociales devient objet de choix et d'élaboration personnelle ${ }^{38}$. De ce fait, «il faut développer, pour les besoins de sa propre survie, une image du monde centrée sur le moi, qui renverse en quelque sorte le rapport entre le moi et la société, et l'adapte aux objectifs de l'organisation individuelle de l'existence $»^{39}$.

Dans cette voie, la sociologie devient victime de la réflexivité que produisent les théories formulées en son nom. En effet, les individus, éclairés par leur lumière, parviendraient de nos jours à «forger leur individualité ${ }^{40}$ et leur position sociale de leur propre chef en risquant de rendre caduque la domination qui s'exerce à l'échelle de la société. Bref, l'éclipse de la domination sociale en vertu de laquelle les sociologues étaient jadis fondés à concevoir l'individu en tant qu'objet de leurs études permet aujourd'hui à ce dernier de devenir sujet au sens où l'entend Touraine de «manifester une conscience de soi-même autofondée» propice à pouvoir se représenter à lui-même «sa propre capacité de création, de réflexion, et d'évaluation».

\footnotetext{
35 Pierre Bourdieu, Méditations pascaliennes, coll. Liber, Paris, Seuil, 1997, p. 277.

${ }^{36}$ Ulrich Beck, La société du risque, Paris, Aubier, 2001, p. 283.

${ }^{37}$ Zygmunt Bauman, La vie liquide, Paris, Le Rouergue / Chambon, 2006, p. 7.

${ }^{38}$ Ulrich Beck, La société du risque, op. cit., p. 290.

${ }^{39}$ Ibid., p. 291.

${ }^{40}$ Danilo Martucelli, Forger par l'épreuve, Paris, Armand Colin, 2006.
} 


\section{EN MANIÈRE DE CONCLUSION LA SOCIOLOGIE FACE À LA DISPARITION DE SON OBJET?}

Le glas a apparemment sonné pour la «sociologie classique»- la théorie de Bourdieu par exemple - puisque son objet disparaît dans ce contexte. La sociologie doit être aujourd'hui « refaite ${ }^{41}$ ou « refondée » afin de tenir compte du fait que les théories formulées sous son égide « vont et viennent» continuellement par rapport à leur objet, selon l'affirmation de Giddens, et contribuent à transformer cet objet en sujet susceptible de comprendre le jeu social et de l'orienter en sa faveur afin d'être lui-même chef de sa "vérité, sa morale, ses liens sociaux et son identité » ${ }^{42}$.

La réflexivité de la sociologie responsable de la disparition progressive de son objet est toutefois sujette à débats chez des auteurs comme Alain Ehrenberg, selon lesquels la société ne peut nullement être considérée «comme un tas d'expériences individuelles reposant sur la subjectivité de chacun». La société semble en effet correspondre strictement à des «relations intersubjectives» en vertu desquelles «l'individu produirait à lui seul, subjectivement et grâce à sa capacité réflexive, le lien social dans ses interactions avec d'autres sujets ${ }^{43}$.

Les artisans des méthodes susceptibles de produire la réflexivité de la sociologie reconnaissent d'autre part, comme François Dubet à propos de l'intervention sociologique, que cette dernière «n'engendre pas forcément d'effets perceptibles et les acteurs concernés retrouvent très rapidement les catégories de l'action ${ }^{44}$ qui, chez Bourdieu, correspondent aux «routines de la pensée ordinaire ». Inversement, «quand les chercheurs sont invités à passer du côté de l'action, à donner des conseils, il n'est pas certain qu'ils aient acquis une compétence particulière ${ }^{45}$. La réflexivité de la sociologie, on le constate sans peine, demeure toute relative. La sociologie se révèle certes une «connaissance d'une connaissance», comme l'affirme pertinemment Bourdieu, c'est-à-dire une connaissance théorique basée sur la connaissance pratique des individus associée au sens commun. Celui-ci, on le devine, apparaît pareillement une «connaissance d'une connaissance», une réflexion ouverte à la connaissance sociologique, mais également aux autres connaissances sociales que sont par exemple la littérature, la philosophie, la science, le journalisme, la culture médiatique et les autres savoirs qui circulent dans l'orbite sociale.

Force est d'admettre que la réflexivité produite par la sociologie trouve son droit et sa mesure en société sous la concurrence de ces autres connaissances sociales susceptibles d'influer sur la réflexion qu'orchestrent les individus à leur niveau. La théorie sociologique est donc sujette au jeu social en fonction duquel, selon les circonstances, elle peut être, dans le meilleur des cas, celui de la «démocratisation de la vie individuelle ${ }^{46}$, une connaissance égale aux autres ou, dans le

${ }^{41}$ Bruno Latour, Changer de société Refaire de la sociologie, coll. Armillaire, Paris, La Découverte, 2006.

${ }^{42}$ Jean-Claude Kaufmann, Ego: pour une sociologie de l'individu, Paris, Nathan, 2001, p. 112.

43 Alain Ehrenberg, «Agir de soi-même», Esprit, no 316, 2005, p. 201.

${ }^{44}$ François Dubet, Sociologie de l'expérience, op. cit., p. 248.

45 Ibid., p. 249.

${ }^{46}$ Jean-Claude Kaufmann, Ego: pour une sociologie de l'individu, op. cit., p. 112. 
pire, dominée par l'une et l'autre de ses concurrentes. Elle peut également contribuer à la domination sociale en vertu du contrôle qui, en prenant la forme de la théorie, ne révèle pas son véritable visage ainsi que l'illustre, aujourd'hui comme hier, l'ingénierie sociale.

La réflexivité de la théorie sociologique doit être conçue tout compte fait avec les nuances apportées jadis par Durkheim selon lequel les sociologues «sont faits pour aider leurs contemporains à se reconnaître dans leurs idées et dans leurs sentiments beaucoup plus que pour les gouverner» et par conséquent «ils s'acquitteront d'autant mieux de leur rôle, qu'ils borneront là leur ambition ${ }^{47}$.

Département de sociologie, Université de Montréal

Case postale 6128, succursale Centre-ville, Montréal, Québec H3C 3J7

Tél.: (514) 343-7159 Fax:(514) 343-5722

Courriel:jacques.hamel@umontreal.ca

\section{BIBLIOGRAPHIE}

Bauman, Zygmunt, La vie liquide, Paris, Le Rouergue / Chambon, 2006.

Beck, Ulrich, La société du risque, Paris, Aubier, 2001.

Bourdieu, Pierre, Méditations pascaliennes, coll. Liber, Paris, Seuil, 1997.

Bourdieu, Pierre, Raisons pratiques, Paris, Seuil, 1994

Bourdieu, Pierre (sous la dir.), La misère du monde, Paris, Seuil, 1993.

Bourdieu, Pierre, Le sens pratique, Paris, Minuit, 1980.

Bourdieu, Pierre, «Introduction», dans Pierre Bourdieu (sous la dir.), Un art moyen. Essai sur les usages sociaux de la photographie, Paris, Editions de Minuit, 1965, p.17-28.

Bourdieu, Pierre (avec la coll. de L. Wacquant) (1992), Réponses, Paris, Seuil, 1992.

Bourdieu, Pierre, Jean-Claude Chamboredon et Jean-Claude Passeron, Le métier de sociologue, Paris, Mouton, 1968.

Dubet, François, «Plaidoyer pour l'intervention sociologique », dans Didier Vrancken et Olgierd Kuty (sous la dir.), La sociologie et l' intervention, Bruxelles, De Boeck, 2001, p. 89-110.

Dubet, François, Sociologie de l'expérience, Paris, Seuil, 1994.

Dubet, François, Acteurs et sociologues. Le cas de l' intervention sociologique, Paris, Ecole des hautes études en sciences sociales, miméographié, 1988.

Durkheim, Emile, «L'élite intellectuelle et la démocratie», Revue bleue, $5^{e}$ série, tome 1, 1904, p. 705-706.

Ehrenberg, Alain, «Agir de soi-même», Esprit, no 316, 2005, p. 200-209.

Giddens, Anthony, La transformation de l' intimité, Paris, Le Rouergue / Chambon, 2004.

Giddens, Anthony, Les conséquences de la modernité, Paris, L'Harmattan, 1994.

Guth, Suzie, Chicago 1920, aux origines de la sociologie qualitative, Paris, Taraèdre, 2004.

Habermas, Jürgen, Idéalisations et communication, Paris, Fayard, 2006.

Habermas, Jürgen, La technique et la science comme «idéologie», Paris, Gallimard, 1973.

Kaufmann, Jean-Claude, Ego: pour une sociologie de l' individu, Paris, Nathan, 2001.

Latour, Bruno, Changer de société Refaire de la sociologie, coll. Armillaire, Paris, La Découverte, 2006.

Martucelli, Danilo, Forger par l'épreuve, Paris, Armand Colin, 2006.

Touraine, Alain, Un nouveau paradigme. Pour comprendre le monde d'aujourd' hui, Paris, Fayard, 2005 .

${ }^{47}$ Emile Durkheim, «L'élite intellectuelle et la démocratie», op. cit., p. 706. 